\title{
Comunicación
}

\section{VALORES HEMATOLÓGICOS REFERENCIALES EN AÑUJES (Dasyprocta fuliginosa) MANTENIDOS EN CAUTIVERIO}

\section{Referential Haematological Values in Agoutis (Dasyprocta fuliginosa) KEPT IN CAPTIVITY}

\author{
Dante Andrade E. ${ }^{1}$, Olga Li E. ${ }^{1,2}$, Francisco Suárez A. ${ }^{3}$, Walter Silva S. ${ }^{4}$
}

\section{Resumen}

\begin{abstract}
El objetivo del presente estudio fue determinar los valores hematológicos del añuje (Dasyprocta fuliginosa) criado en cautiverio en el Zoológico Patronato Parque Las Leyendas (PATPAL). Se utilizaron 23 animales (11 machos y 12 hembras; 13 juveniles y 10 adultos). Los animales fueron anestesiados con una combinación de Ketamina (10 mg/kg peso vivo) y Xilacina (1 mg/kg peso vivo) i.m. El análisis hematológico incluyó hemoglobina $(\mathrm{Hb})$, hematocrito $(\mathrm{Ht})$, contaje de eritrocitos y leucocitos, Volumen Corpuscular Medio (VGM), Concentración de Hemoglobina Corpuscular Media (CHGM) y Hemoglobina Corpuscular Media (HCM), y el diferencial de leucocitos. Los resultados fueron: $6.17 \times 10^{6} / \mu$ l eritrocitos, $5,739 \times 10^{3} / \mu 1$ leucocitos, $40.4 \% \mathrm{Ht}, 12.42 \mathrm{~g} / \mathrm{dl} \mathrm{Hb}, 66.56 \mathrm{fl} \mathrm{VGM}$, 20.56 pg HCM, 30.88 g/dl CHGM; y $67.1 \%$ neutrófilos, $29.6 \%$ linfocitos, $2.39 \%$ eosinófilos, $0.26 \%$ monocitos y $0.56 \%$ abastonados. Los valores encontrados son similares a otros valores reportados en la literatura.
\end{abstract}

Palabras clave: añuje, Dasyprocta fuliginosa, valores hematológicos, cautiverio, zoológico

\section{Abstract}

The purpose of the present study was to determine the haematologic values of agoutis (Dasyprocta fuliginosa) bred in captivity at the Patronato Parque Las Leyendas zoo (PATPAL). Twenty three animals were used (11 males and 12 females; 13 juvenile and 10 adults). They were anesthetized with a combination of Ketamine $(10 \mathrm{mg} / \mathrm{kg} \mathrm{BW})$ and Xilacine (1 mg/kg BW) i.m. The haemathological analysis included haemoglobin ( $\mathrm{Hb})$, hematocrit (Ht), count of erythrocytes (RBC) and leucocytes (WBC), mean cell volume $(\mathrm{MCV})$, mean corpuscular haemoglobin $(\mathrm{MCH})$, mean corpuscular haemoglobin

\footnotetext{
${ }^{1}$ Laboratorio de Patología Clínica y Biología Molecular, ${ }^{3}$ Laboratorio de Medicina Veterinaria Preventiva, Facultad de Medicina Veterinaria, Universidad Nacional Mayor de San Marcos, Lima

${ }^{2}$ E-mail: olgalie@hotmail.com

${ }^{4}$ Instituto Nacional de Salud (INS), Lima
} 
concentration (MCHC), and the leukocyte differential. The obtained values were: $6.17 \mathrm{x}$ $106 / \mu 1 \mathrm{RBC}, 5,739 \times 10^{3} / \mu \mathrm{l} \mathrm{WBC}, 40.4 \% \mathrm{Ht}, 12.42 \mathrm{~g} / \mathrm{dl} \mathrm{Hb}, 66.56 \mathrm{fl} \mathrm{MCV}, 20.56 \mathrm{pg} \mathrm{MCH}$, $30.88 \mathrm{~g} / \mathrm{dl} \mathrm{MCHC}$; and $67.1 \%$ neutrophils, $29.6 \%$ lymphocytes, $2.39 \%$ eosinophils, $0.26 \%$ monocytes and $0.56 \%$ band neutrophils. The encountered values were similar to those reported in the literature.

Key words: agouti, Dasyprocta fuliginosa, hematological values, captivity, zoo

La fauna silvestre contribuye a la alimentación humana del país, especialmente en la selva, donde se estima un consumo de 13,000 TM de carne de monte. El añuje Dasyprocta fuliginosa) constituye parte importante de la dieta del poblador rural de la región amazónica, en particular cuando escasean los animales grandes (Brack, 1987). En cautiverio, estos animales puede alcanzar un peso de $7 \mathrm{~kg}$, con un rendimiento de canal del 70\% (Ojasti, 1993). La eficiencia productiva y valor nutricional de la carne del añuje comparado con el bovino y otras especies tradicionales es aparentemente superior (Negret, 1984). Además, las vísceras, sangre y huesos se utilizan para la preparación de potajes de la zona, o son empleados para la elaboración de alimentos balanceados para otros animales (Tapia, 1997).

Las investigaciones en animales de fauna silvestre están más orientadas a otras especies de mamíferos, por lo que no se dispone de estudios sobre los valores hematológicos en el añuje. Esta información es de gran utilidad como herramienta auxiliar en el diagnóstico de un individuo clínicamente enfermo, así como para detectar enfermedades subclínicas cuando se realizan los controles sanitarios de rutina. El objetivo del presente trabajo fue determinar los valores hematológicos en añujes clínicamente sanos, mantenidos en cautiverio bajo el mismo manejo en un zoológico de la ciudad de Lima.

El estudio, de tipo descriptivo, se realizó en el Zoológico Patronato Parque Las Leyendas (PATPAL), ubicado en el distrito de San Miguel, Lima, durante el mes de noviembre de 2004. La zona tiene una temperatura mínima de $12.5^{\circ} \mathrm{C}$ y una máxima de $14.5^{\circ} \mathrm{C}$, y una humedad relativa mínima de 54.3 y máxima de $93.5 \%$. El procesamiento de las muestras se llevó a cabo en el Laboratorio de Patología Clínica de la Facultad de Medicina Veterinaria, Universidad Nacional Mayor de San Marcos, Lima.

Se evaluaron 23 animales (13 juveniles y 10 adultos, 12 hembras y 11 machos), que estuvieron bajo las mismas condiciones de manejo, alimentación, sanidad, y ubicados en ambientes similares. La comprobación del estado de salud se hizo mediante la observación del comportamiento y el examen clínico; asimismo, se realizó una evaluación de las constantes fisiológicas (temperatura, frecuencia cardiaca y frecuencia respiratoria).

Para la toma de las muestras, los animales fueron atrapados con redes y trasladados al Hospital Veterinario del PATPAL. Se les anestesió con clorhidrato de ketamina al $10 \%$ en dosis de $10 \mathrm{mg} / \mathrm{kg}$ de peso vivo y Xilacina al $2 \%$ en dosis de $1 \mathrm{mg} / \mathrm{kg}$ de peso vivo, por vía intramuscular (Fowler y Miller, 2000). La sangre se tomó de la vena femoral en tubos tipo vacutainers de $3 \mathrm{ml}$ con anticoagulante EDTA.

Se determinó el Volumen del Paquete Celular o Hematocrito (\%) por el método del microhematocrito, la hemoglobina $(\mathrm{g} / \mathrm{dl})$ por el método de la cianometahemoglobina, el número de glóbulos rojos (x 10\%/ 1 ) (Benjamín, 1991) y glóbulos blancos (x $\left.10^{3} / \mu \mathrm{l}\right)$, el diferencial leucocitario (\%), y los índices eritrocíticos de Volumen Corpuscular Medio (VGM, fl), Concentración de Hemoglobina Corpuscular Media (CHGM, g/dl) y Hemoglobina Corpuscular Media (HCM, pg). Las 
Cuadro 1. Valores de la serie eritrocítica en 23 añujes (Dasyprocta fuliginosa) mantenidos en cautiverio en el Zoológico Patronato Parque de las Leyendas, Lima

\begin{tabular}{lcccc}
\hline & $\begin{array}{c}\text { Valores } \\
\text { Mínimos }\end{array}$ & $\begin{array}{c}\text { Valores } \\
\text { Máximos }\end{array}$ & Media & d.e. $^{1}$ \\
\hline $\begin{array}{l}\text { Glóbulos rojos (x } \\
\left.10^{6} / \mathrm{\mu l}\right)\end{array}$ & 4.860 & 9.180 & 6.174 & 0.973 \\
Hematocrito (\%) & 35 & 46.0 & 40.3 & 2.7 \\
$\mathrm{Hemoglobina}(\mathrm{g} / \mathrm{dl})_{\mathrm{VCM}^{2}(\mathrm{fl})}$ & 10.8 & 14.4 & 12.4 & 0.9 \\
$\mathrm{CHCM}^{3}(\mathrm{~g} / \mathrm{dl})$ & 47.9 & 88.6 & 66.6 & 10.0 \\
$\mathrm{HCM}^{4}(\mathrm{pg})$ & 27.8 & 34.2 & 30.9 & 1.9 \\
\hline
\end{tabular}

${ }^{1}$ Desvío estándar

${ }^{2}$ Volumen Corpuscular Medio

${ }^{3}$ Concentración de Hemoglobina Corpuscular Media

${ }^{4}$ Hemoglobina Corpuscular Media

Cuadro 2. Valores de la serie leucocítica en 23 añujes (Dasyprocta fuliginosa) mantenidos en cautiverio en el Zoológico Patronato Parque de las Leyendas, Lima

\begin{tabular}{lcccc}
\hline & $\begin{array}{c}\text { Valores } \\
\text { Mínimos }\end{array}$ & $\begin{array}{c}\text { Valores } \\
\text { Máximos }\end{array}$ & Media & d.e. $^{1}$ \\
\hline Leucocitos & 3200 & 10400 & 5739 & 1810 \\
Abastonados & 0 & 3 & 0.57 & 0.90 \\
Neutrófilos & 42 & 87 & 67.09 & 10.79 \\
Linfocitos & 8 & 58 & 29.65 & 12.20 \\
Eosinófilos & 0 & 15 & 2.39 & 3.62 \\
Monocitos & 0 & 2 & 0.26 & 0.541 \\
\hline
\end{tabular}

${ }^{1}$ Desvío estándar

constantes hematológicas fueron obtenidas, según los protocolos tomados de Benjamín (1991).

En el análisis estadístico de los parámetros hematológicos y las constantes fisiológicas se emplearon la media aritmética como medida de tendencia central y la desviación estándar como medida de dispersión para expresar los resultados encontrados. Para determinar diferencia estadística entre los parámetros hematológicos y las constantes fisiológicas por efecto del sexo y la edad (1 a 3 años y 5 a 16 años) se empleó la prueba de $\mathrm{T}$ de Student para muestras independientes.

Los resultados de la serie eritrocítica y leucocítica se muestran en los cuadros 1 y 2. No se encontró diferencia estadística por efecto del sexo o la edad, aunque se pudo apreciar valores ligeramente superiores en los machos para hematocrito y número de glóbulos rojos; posiblemente debido al efec- 
to de la testosterona, que estimula el aumento en la producción de eritropoyetina, por medio de la trascripción mediada por receptores y por efecto directo sobre la medula ósea (García, 1995). Así mismo, los adultos registraron promedios ligeramente más altos en la serie eritrocítica. La temperatura, frecuencia cardiaca y frecuencia respiratoria no estuvieron influenciadas por efecto del sexo, edad y peso.

Los valores encontrados en la serie eritrocitica son similares a los reportados por ISIS (1998, 2002), con excepción del hematocrito donde se reportan valores de $45 \%$. Similarmente, los resultados de la serie leucocítica fueron bastante similares a lo encontrados en estos trabajos con excepción del porcentaje de monocitos donde se repor$\tan$ valores superiores al 5\%.

Se concluye que los resultados guardan estrecha relación con otros reportes de la misma especie, y por tanto pueden ser considerados como patrones referenciales de la especie, en condiciones de manejo de crianza en cautiverio.

\section{Literatura Citada}

1. Benjamín M. 1991. Manual de patología clínica. $3^{a}$ ed. México DF: Ed. Limusa. 421 p.
2. Brack A. 1987. La fauna. En: Manfer, Mejía Baca J (eds). Gran geografía del Perú: naturaleza y hombre. Vol. III. España: LIMUSA. 247 p.

3. Fowler E, Miller E. 2000. Zoo \& wild animal medicine: Current therapy $4^{\text {th }}$ ed. Denver, Colorado: WB Saunders. 617 p.

4. García S. 1995. Fisiología veterinaria. Madrid: Ed. Interamericana. 1074 p.

5. [ISIS] Internacional Species Information System. 1998. Valores Hematológicos de Dasyprocta leonina [Internet], [2 junio 2005] Disponible en: http://www.isis.org.com

6. [ISIS] Internacional Species Information System. 2002. Valores hematológicos de Dasyprocta leonina [Internet], [2 junio 2005] Disponible en: http://www.isis.org.com

7. Negret R. 1984. Ecología y manejo de la fauna silvestre. Bogotá: Corporación de Araracuara. $154 \mathrm{p}$.

8. Ojasti J. 1993. Utilización de la fauna silvestre en América Latina. Situación y perspectivas para un manejo sostenible. Guías FAO: Conservación-25. Roma: FAO. 265 p.

9. Tapia M. 1997. Guía para el manejo y cría del aguti, picure, guatusa o ñeque Dasyprocta punctata Gray y Dasyprocta fuliginosa Wagler. Santa Fé de Bogotá: Convenio Andrés Bello. 46 p. 